\title{
The prognosis and risk factors for acute kidney injury in high-risk patients after surgery for type A aortic dissection in the ICU
}

\author{
Kun Zhang ${ }^{1 \#}$, Jiuyan Shang ${ }^{2 \#}$, Yuhong Chen ${ }^{1 \#}$, Yan Huo ${ }^{1 \#}$, Bin Li $^{1 \#}$, Zhenjie $\mathrm{Hu}^{1}$ \\ ${ }^{1}$ Intensive Care Unit, Hebei Medical University Fourth Affiliated Hospital and Hebei Provincial Tumor Hospital, Shijiazhuang, China; ${ }^{2}$ Department \\ of Pathology, Hebei Medical University Fourth Affiliated Hospital and Hebei Provincial Tumor Hospital, Shijiazhuang, China \\ Contributions: (I) Conception and design: K Zhang, Y Chen, Z Hu; (II) Administrative support: None; (III) Provision of study materials or patients: K \\ Zhang; (IV) Collection and assembly of data: K Zhang, J Shang; (V) Data analysis and interpretation: K Zhang, J Shang; (VI) Manuscript writing: All \\ authors; (VII) Final approval of manuscript: All authors. \\ \#These authors contributed equally to this work. \\ Correspondence to: Prof. Zhenjie Hu, MD. Intensive Care Unit, Hebei Medical University Fourth Affiliated Hospital and Hebei Provincial Tumor \\ Hospital, Shijiazhuang, China. Email: syicu@vip.sina.com.
}

Background: Acute kidney injury (AKI) is a major complication of cardiac surgery, with high rates of morbidity and mortality. The aim of this study was to identify risk factors for the incidence and prognosis of AKI in high-risk patients before and after surgery for acute type A aortic dissection (TAAD) in the intensive care unit (ICU).

Methods: We performed a retrospective cohort study from April 2018 to April 2019. The primary end points of this study were morbidity due to AKI and risk factors for incidence, and the secondary end points were mortality at 28 days and risk factors for death.

Results: We enrolled 60 patients, 52 (86.67\%) patients developed postoperative AKI, 28 (53.84\%) patients died. Preoperative lactic acid level $(\mathrm{P}=0.022)$ and cardiopulmonary bypass $(\mathrm{CPB})$ duration $(\mathrm{P}=0.009)$ were identified as independent risk factors for postoperative AKI. The 28-day mortality for postoperative patients with TAAD was $46.67 \%$, 53.84\% for those with TAAD and AKI, $67.5 \%$ for those who required continue renal replacement therapy (CRRT). The risk factors for 28-day mortality due to postoperative AKI for patients requiring CRRT were $\mathrm{CPB}$ duration $(\mathrm{P}=0.019)$ and norepinephrine dose upon diagnosis of AKI $(\mathrm{P}=0.037)$.

Conclusions: Morbidity due to AKI in postoperative patients with TAAD was $86.67 \%$, and preoperative lactic acid level and CPB duration were independent risk factors. The 28-day mortality of postoperative patients with TAAD was $46.67 \%, 53.84 \%$ for those with TAAD and AKI, and $67.5 \%$ for those requiring CRRT. CPB duration and norepinephrine dose upon diagnosis of AKI may influence patients' short-term prognosis.

Keywords: Intensive care unit (ICU); acute kidney injury (AKI); type A aortic dissection (TAAD); continue renal replacement therapy (CRRT)

Submitted Feb 03, 2021. Accepted for publication Jul 13, 2021.

doi: $10.21037 /$ jtd-21-823

View this article at: https://dx.doi.org/10.21037/jtd-21-823

\section{Introduction}

Acute kidney injury (AKI) is a major complication and an independent risk factor for high morbidity and mortality in critically ill patients $(1,2)$. According to previous studies, the prevalence of AKI after cardiac surgery ranges from
$19.3 \%$ to $54 \%(3-5)$, which was higher than that reported for type A aortic dissection (TAAD) patients (6). Insufficient tissue perfusion, cellular oxygenation, and heart dysfunction may be the leading causes of this kind of kidney injury (7). Other studies have shown that high body mass index (BMI), 


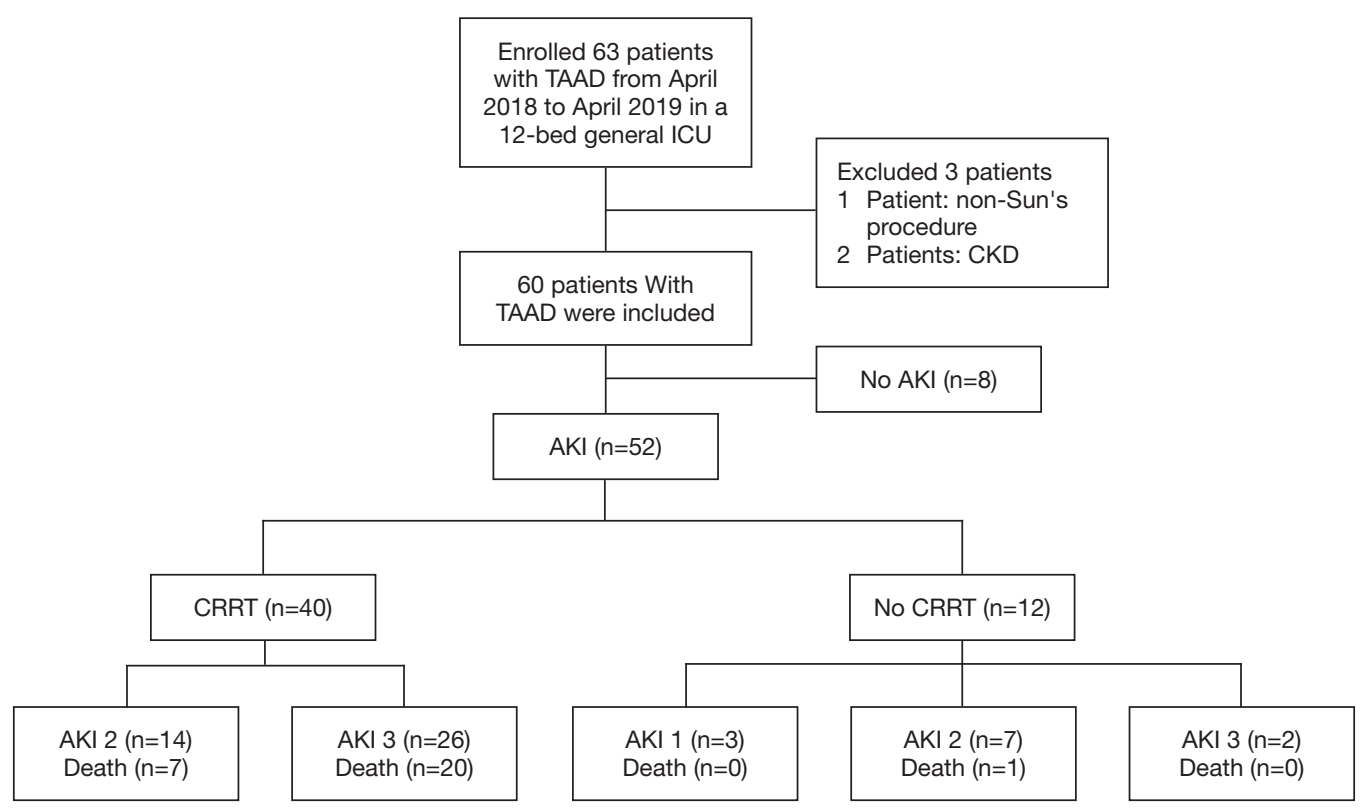

Figure 1 Flowchart of study participants. TAAD, type A aortic dissection; CKD, chronic kidney disease; ICU, intensive care unit; CRRT, continue renal replacement therapy.

advanced age, perioperative peak serum C-reactive protein (CRP) concentration, perioperative sepsis, preexisting renal impairment, and cardiopulmonary bypass time were independent risk factors for postoperative AKI (6,8-10). Recent data has also revealed that postoperative AKI is associated with increased rates of morbidity, cost, and mortality at 30 or 90 days $(3,11,12)$.

By regulating fluid balance, acid-base homeostasis, and electrolyte disorder, continue renal replacement therapy (CRRT) has been considered an effective therapy for AKI $(13,14)$. However, the survival of patients with AKI remains low (15-17), with mortality rates of $12.2 \%$ for none renal replacement therapy (non-RRT) AKI and $46.9 \%$ for RRTAKI patients (18). Previous studies have documented that approximately $2-9 \%$ of TAAD patients require CRRT after surgery, and the mortality of this cohort is up to $64 \%(6,9,19)$.

Most of the aforementioned data were obtained from general patients after cardiothoracic surgery. However, critically ill patients who were transferred to intensive care unit (ICU) after TAAD surgery had relatively severe illness, and the incidence, outcomes, and risk factors for postoperative AKI were unknown. In our daily work, we observed a high incidence of AKI and poor prognosis in patients who underwent cardiac surgery, especially in those with TAAD. Therefore, we designed a retrospective study to explore the risk factors for developing AKI as well as its prognostic factors in patients who underwent surgery for TAAD. This study aimed to provide some guidance to clinicians for improving the outcomes of patients with TAAD. We present the following article in accordance with the STROBE reporting checklist (available at https://dx.doi. org/10.21037/jtd-21-823).

\section{Methods}

\section{Design and setting}

This retrospective cohort study initially enrolled 63 patients with TAAD in a 12-bed general ICU in the Fourth Hospital of Hebei Medical University from April 2018 to April 2019 (Figure 1). The exclusion criteria were as follows: (I) patients younger than 18 years; (II) patients undergoing chronic dialysis due to chronic kidney disease (CKD); and (III) patients that did not undergo Sun's procedure for TAAD (20). Finally, 60 patients were included (two patients were excluded due to of CKD, and one patient was excluded as they did not undergo Sun's procedure). AKI was defined by the Kidney Disease Improving Global Outcomes (KDIGO) criteria (Table 1) (21). This study was approved by Ethics Committee of the Fourth Hospital of Hebei Medical University (2017MEC106), and complied with the Helsinki Declaration guidelines (as revised in 2013). All patients 
Table 1 Kidney Disease Improving Global Outcomes (KDIGO) criteria for AKI

\begin{tabular}{lll}
\hline Stage & Serum creatinine increase & Urine output decrease \\
\hline 1 & $1.50-1.90$ times baseline or $\geq 0.30 \mathrm{mg} / \mathrm{dL}(26.50 \mu \mathrm{mol} / \mathrm{L})$ increase & Urine output $<0.5 \mathrm{~mL} / \mathrm{kg} / \mathrm{h}$ for $6-12 \mathrm{hours}$ \\
2 & $2.00-2.90$ times baseline & Urine output $<0.5 \mathrm{~mL} / \mathrm{kg} / \mathrm{h}$ for $\geq 12 \mathrm{hours}$ \\
3 & $\geq 3.00$ times baseline or increase in serum creatinine to $\geq 4.00 \mathrm{mg} / \mathrm{dL}$ & Urine output $<0.3 \mathrm{~mL} / \mathrm{kg} / \mathrm{h}$ for $\geq 24 \mathrm{hours}$ or \\
& $(353.60 \mu \mathrm{mol} / \mathrm{L})$ or initiation of renal replacement therapy & Anuria for $\geq 12$ hours \\
\hline
\end{tabular}

$\mathrm{AKI}$, acute kidney injury.

signed the informed consent at admission.

\section{Data collection}

We collected preoperative demographic parameters including age, gender, baseline serum creatinine $(\mathrm{sCr})$, blood urea nitrogen (BUN), systolic blood pressure (SBP), diastolic blood pressure (DBP), mean arterial pressure (MAP), heart rate (HR), respiratory rate (RR), percutaneous arterial oxygen saturation $\left(\mathrm{SpO}_{2}\right)$, body temperature $(\mathrm{T})$, and central venous pressure (CVP). The APACHE II score was recorded upon admission to the ICU, and laboratory results were recorded at the time of AKI diagnosis. We also calculated the estimated glomerular filtration rate (eGFR) using the Modification of Diet in Renal Disease (MDRD) equation (22). Daily fluid balance and CVP in the ICU were reviewed. We also recorded positive end expiratory pressure (PEEP) and inspired oxygen concentration $\left(\mathrm{FiO}_{2}\right)$ for patients who used a ventilator.

\section{Statistical analyses}

Data were analyzed with SPSS 22.0 (SPSS IBM Corp., Armonk, NY, USA). All statistics were expressed as mean \pm standard for continuous variables. The Student's $t$-test was used to compare continuous variables, and the chi squared $\left(\chi^{2}\right)$ or Fisher exact tests was used to compare categorical variables of different groups. Logistic regression models were used to identify univariate and multivariate risk factors for AKI and mortality of patients undergoing CRRT. The multivariate model included variables that were significant in the univariate analysis. All statistical tests were two-sided and $\mathrm{P}<0.05$ was considered statistically significant.

\section{Results}

\section{Patient characteristics}

The mean age of the 60 included patients was
$54.95 \pm 13.81$ years (range, $28-78$ years), and $43(71.67 \%)$ were men. Previous medical histories of the patients included hypertension $(75.00 \%)$, diabetes $(5.00 \%)$, coronary heart disease $(8.33 \%)$, cerebrovascular disease $(16.67 \%)$, and chronic obstructive pulmonary disease $(3.33 \%)$. The mean durations of the operation, cardiopulmonary bypass (CPB), and aortic cross-clamping (ACC) were 8.00 (6.50, 9.05) hours, $171.00(151.50,196.25)$ minutes, and 107.00 $(81.50,126.75)$ minutes, respectively. The baseline sCr was $79.38 \pm 23.19 \mathrm{mmol} / \mathrm{L}$ (Table 2).

\section{Incidence and risk factors for postoperative AKI}

According to the KDIGO criteria, $52(86.67 \%)$ patients developed postoperative AKI; 3 patients (5.00\%) were in stage 1, 21 patients $(35.00 \%)$ were in stage 2 , and 28 patients $(46.70 \%)$ were in stage 3 . The risk factors for postoperative AKI are shown (Table 3). The identified risk factors for postoperative AKI were preoperative lactic acid level [odds ratio (OR), 1.409; 95\% confidence interval (CI), 1.051-1.890; $\mathrm{P}=0.022]$ and $\mathrm{CPB}$ duration $(\mathrm{OR}, 1.024 ; 95 \%$ CI, 1.006-1.042; $\mathrm{P}=0.009$ ) (Table 4).

\section{Outcomes of the postoperative patients}

CRRT was required in $40(66.67 \%)$ patients with AKI; of these, $14(23.33 \%)$ patients were in stage 2 and half of them died, while $26(43.34 \%)$ patients were in stage 3 , and $76.92 \%$ of them died. The CRRT patients were divided into two groups: a survival group and a non-survival group. Univariate analysis (Table 5) found six variables that were associated with the 28-day mortality of patients with postoperative AKI requiring CRRT. Multivariate analysis revealed that the risk factors for 28-day mortality in patients with postoperative AKI requiring CRRT were CPB duration (OR, 1.037; 95\% CI, 1.006-1.068; $\mathrm{P}=0.019$ ) and norepinephrine dose upon diagnosis of AKI (OR, 1.523; 
Table 2 Patient characteristics

\begin{tabular}{|c|c|}
\hline Variables & Value $(n=60)$ \\
\hline \multicolumn{2}{|l|}{ Demographic data } \\
\hline Age (year) & $54.95 \pm 13.81$ \\
\hline Male (\%) & $43(71.67)$ \\
\hline \multicolumn{2}{|l|}{ Medical history, n (\%) } \\
\hline Hypertension & $45(75.00)$ \\
\hline Diabetes & $3(5.00)$ \\
\hline Coronary heart disease & $5(8.33)$ \\
\hline COPD & 2 (3.33) \\
\hline Cerebrovascular disease & $10(16.67)$ \\
\hline \multicolumn{2}{|l|}{ Preoperative condition } \\
\hline Temperature $\left({ }^{\circ} \mathrm{C}\right)$ & $36.5(36.1,36.7)$ \\
\hline Heart rate $(\mathrm{bpm})$ & $80(71,88)$ \\
\hline Respiration rate (bpm) & $19(17,20)$ \\
\hline Systolic blood pressure $(\mathrm{mmHg})$ & $133 \pm 29$ \\
\hline Diastolic blood pressure (mmHg) & $76 \pm 16$ \\
\hline Leukocyte (×10\%/L) & $11.91 \pm 3.62$ \\
\hline Hemoglobin (g/L) & $130.05(121.45,141.75)$ \\
\hline Platelet $\left(\times 10^{6} / \mathrm{L}\right)$ & $169.90 \pm 51.21$ \\
\hline Blood urea nitrogen (mmol/L) & $6.30(4.98,7.83)$ \\
\hline Baseline serum creatinine $(\mu \mathrm{mol} / \mathrm{L})$ & $79.38 \pm 23.19$ \\
\hline Troponin I (ng/mL) & $0.06(0.01,1.91)$ \\
\hline Lactic acid (mmol/L) & $2.30(1.70,4.65)$ \\
\hline \multicolumn{2}{|l|}{ Operative details } \\
\hline Duration of operative (h) & $8.00(6.50,9.05)$ \\
\hline Duration of CPB (min) & $171.00(151.50,196.25)$ \\
\hline Duration of ACC (min) & $107.00(81.50,126.75)$ \\
\hline \multicolumn{2}{|l|}{ Postoperative condition } \\
\hline APACHE II & $19(17,24)$ \\
\hline
\end{tabular}

The data are shown as $\mathrm{n}(\%)$ or median (IQR) or mean \pm SD. CPB, cardiopulmonary bypass; ACC, aortic cross-clamping; APACHE II, Acute Physiology and Chronic Health Evaluation II.

95\% CI, 1.026-2.261; $\mathrm{P}=0.037$ ) (Table 6).

\section{Discussion}

In this retrospective study, the incidence and risk factors for
AKI were investigated in patients who were transferred to the ICU after surgery for TAAD. Moreover, the prognosis and factors that influenced the 28-day mortality were evaluated. The results showed that $86.67 \%$ of patients developed postoperative AKI, and $66.67 \%$ of them required CRRT. The 28-day mortality in the postoperative patients with TAAD was $46.67 \%$, which increased to up to $53.84 \%$ in those who developed AKI, and to $67.50 \%$ in those who required CRRT. The independent risk factors for postoperative AKI were preoperative lactic acid level and $\mathrm{CPB}$ duration, and the independent risk factors for 28-day mortality in those who developed AKI and required CRRT were norepinephrine dose upon diagnosis of $\mathrm{AKI}$ and $\mathrm{CPB}$ duration.

Previous studies have reported the incidence and risk factors for postoperative AKI in cardiovascular surgery departments $(6,23,24)$. Ko et al. (6) reported that the incidence of AKI in TAAD patients was $44 \%$, and only $9 \%$ patients required CRRT. In that study, CPB, high BMI, perioperative peak serum CRP concentration, renal malperfusion, and perioperative sepsis were identified as independent risk factors for postoperative AKI. However, Englberger et al. (25) reported a lower incidence of AKI (17.7\%) and RRT (2.1\%) in patients who had undergone elective thoracic aortic surgery. The reason for this discordance might be the exclusion of emergency surgery and TAAD patients. In one recent meta-analysis (8), the average incidence of AKI in TAAD was $46.3 \%$, and a relatively high morbidity of $66.7 \%$ was observed in overweight patients. Their data demonstrated that high BMI, advanced age, and perioperative sepsis were the independent risk factors for postoperative AKI. Compared with the previous research, our study showed a higher incidence of AKI. This difference may be partly explained by our different target population, which included only patients who underwent Sun's procedure for TAAD, in order to minimize the confounding factors. In addition, compared with the patient population studied in cardiovascular surgery departments, those transferred to the ICU had relatively severe illnesses; for this reason, surgeons made a wise decision to transfer these patients to the ICU.

According the results of previous studies, the preoperative risk factors for developing AKI after cardiac surgery were advanced age, female sex, hypertension, hyperlipidemia, CKD, liver disease, peripheral vascular disease, previous stroke, smoking history, diabetes, and anemia (26). In this study, we collected as many risk factors as possible in order to determine the risk factors 
Table 3 Patient characteristics

\begin{tabular}{|c|c|c|c|}
\hline Variables & AKI $(n=52)$ & No AKI $(n=8)$ & $\mathrm{P}$ \\
\hline Age (year) & $55.15 \pm 13.88$ & $53.63 \pm 14.15$ & 0.773 \\
\hline Male (\%) & $37(71.15)$ & $6(75.00)$ & 0.740 \\
\hline \multicolumn{4}{|l|}{ Medical history, n (\%) } \\
\hline Diabetes & $3(5.77)$ & $0(0.00)$ & 1.000 \\
\hline Coronary heart disease & $5(9.61)$ & $0(0.00)$ & 1.000 \\
\hline COPD & $1(1.92)$ & $1(12.50)$ & 0.251 \\
\hline Cerebrovascular disease & $9(17.31)$ & $1(12.50)$ & 1.000 \\
\hline Respiration rate (bpm) & $19(17,21)$ & $18(17,19)$ & 0.373 \\
\hline Systolic blood pressure $(\mathrm{mmHg})$ & $135 \pm 29$ & $116 \pm 18$ & 0.091 \\
\hline Diastolic blood pressure $(\mathrm{mmHg})$ & $76 \pm 15$ & $68 \pm 15$ & 0.183 \\
\hline Leukocyte $\left(\times 10^{9} / \mathrm{L}\right)$ & $12.13 \pm 3.69$ & $10.50 \pm 2.86$ & 0.238 \\
\hline Hemoglobin (g/L) & $130.10(122.65,143.28)$ & $121.70(107.58,136.83)$ & 0.414 \\
\hline Platelet $\left(\times 10^{6} / \mathrm{L}\right)$ & $170.19 \pm 49.31$ & $168.00 \pm 66.20$ & 0.912 \\
\hline Blood urea nitrogen (mmol/L) & $6.60(5.40,7.93)$ & $4.75(4.60,6.03)$ & 0.569 \\
\hline Duration of CPB (min) & $175.50(152.00,206.00)$ & $161.00(145.5,175.00)$ & 0.004 \\
\hline Duration of ACC (min) & $106.00(78.75,128.50)$ & $112.50(103.25,125.75)$ & 0.992 \\
\hline \multicolumn{4}{|l|}{ Postoperative condition } \\
\hline APACHE II & $20(18,24)$ & $17(14,21)$ & 0.030 \\
\hline
\end{tabular}

The data are shown as $\mathrm{n}(\%)$ or median (IQR) or mean \pm SD. AKI, acute kidney injury; COPD, chronic obstructive pulmonary disease; CPB, cardiopulmonary bypass; ACC, aortic cross-clamping; APACHE II, Acute Physiology and Chronic Health Evaluation II.

Table 4 Multivariate analysis of risk factors for postoperative AKI

\begin{tabular}{lcccc}
\hline Variables & OR & $95 \% \mathrm{Cl}$ & $\mathrm{P}$ \\
\hline Hypertension & 0.413 & $0.064-2.654$ & $1.051-1.890$ & 0.351 \\
Lactic acid & 1.409 & $0.806-1.464$ & 0.022 \\
Duration of operative & 1.086 & $1.006-1.042$ & 0.588 \\
Duration of CPB & 1.024 & 0.009 \\
\hline
\end{tabular}

AKI, acute kidney injury; CPB, cardiopulmonary bypass. 
Table 5 Univariate analysis for 28-day mortality of postoperative AKI with CRRT

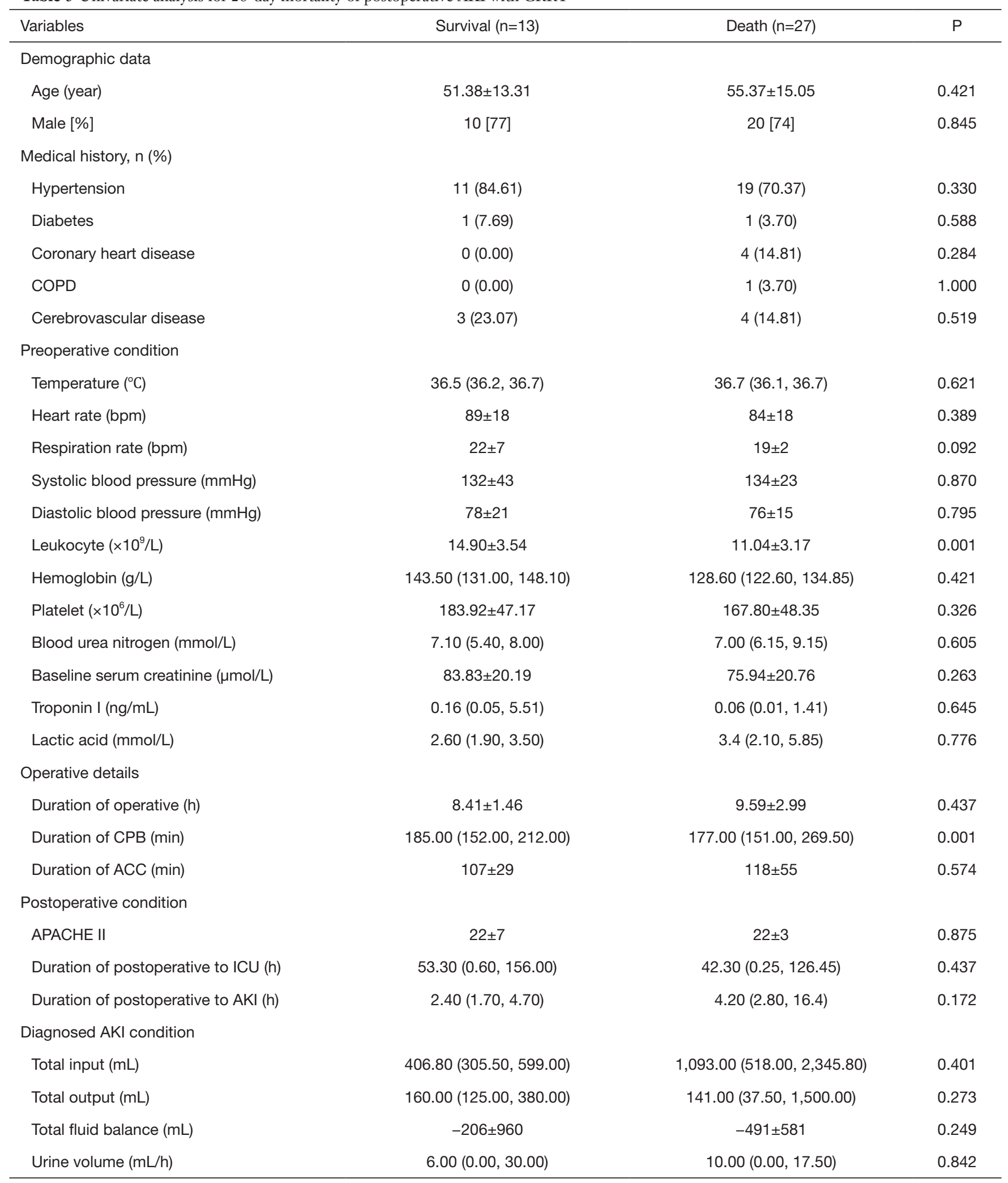

Table 5 (continued) 
Table 5 (continued)

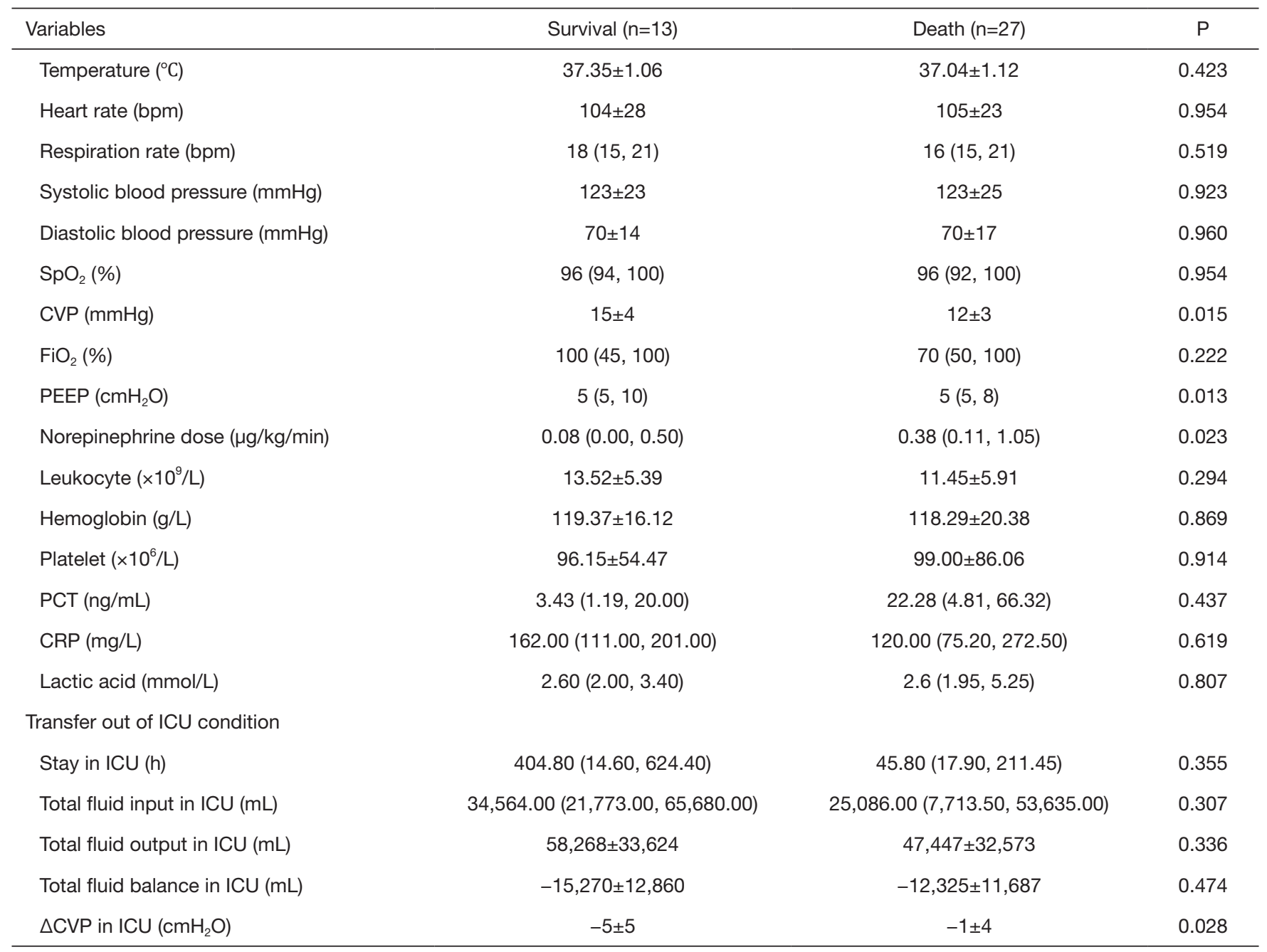

The data are shown as $n(\%)$ or median (IQR) or mean \pm SD. CRRT, continue renal replacement therapy; COPD, chronic obstructive pulmonary disease; CPB, cardiopulmonary bypass; ACC, aortic cross-clamping; APACHE II, Acute Physiology and Chronic Health Evaluation II; ICU, intensive care unit; $\mathrm{AKI}$, acute kidney injury; $\mathrm{SpO}_{2}$, pulse oxygen saturation; $\mathrm{CVP}$, central venous pressure; $\mathrm{FiO}{ }_{2}$, fraction of inspiration $\mathrm{O}_{2}$; PEEP, positive end expiratory pressure; PCT, procalcitonin; CRP, C-reactive protein.

Table 6 Multivariate analysis for 28-day mortality of postoperative AKI with CRRT

\begin{tabular}{lccc}
\hline Variables & OR & $95 \% \mathrm{Cl}$ & P \\
\hline Preoperative leukocyte & 0.470 & $0.208-1.064$ & 0.070 \\
Duration of CPB & 1.037 & $1.006-1.068$ & 0.019 \\
CVP of diagnosed AKI & 0.731 & $0.516-1.034$ & 0.077 \\
PEEP of diagnosed AKI & 0.695 & $0.469-1.029$ & 0.069 \\
Norepinephrine dose of diagnosed AKI & 1.523 & $1.026-2.261$ & 0.037 \\
$\triangle$ CVP in ICU & 1.367 & $0.750-2.494$ & 0.308 \\
\hline
\end{tabular}

CRRT, continue renal replacement therapy; CPB, cardiopulmonary bypass; CVP, central venous pressure; AKI, acute kidney injury; ICU, intensive care unit. 
for postoperative AKI in patients with TAAD. Our results showed that hypertension, preoperative lactic acid, operative duration, CPB duration, and APACHE II score may be the risk factors for postoperative AKI in patients with TAAD. Further analyses identified preoperative lactic acid level as one of the independent risk factors for postoperative AKI in patients with TAAD; this had been seldom reported in previous studies. Lactic acid level is frequently used in clinical practice to reflect tissue perfusion and disease severity. We noted a correlation between insufficient tissue perfusion and perioperative complications, as in the published reports, which mentioned that $16-33 \%$ of cases with TAAD presented with visceral hypoperfusion $(27,28)$. Preoperative lactic acid level was cited as an indicator of malperfusion. We surmised that the degree of laceration was one of the causes of increased lactic acid level during the preoperative preparation for TAAD surgery. In addition, there might have been problems in the fluid management from the onset of aortic dissection to surgery. The influence of these factors for hypoperfusion may have resulted in postoperative AKI. This result reminded us to pay more attention to hemodynamic monitoring and tissue perfusion during the preoperative preparation for cardiac surgery, as this might prevent renal insufficiency and reduce the risk of postoperative AKI in patients with TAAD.

In this study, another independent risk factor for postoperative AKI in patients with TAAD was $\mathrm{CPB}$ duration, which was consistent with current literature. $\mathrm{CPB}$ can result in systemic inflammation and oxidant stress response, which had been generally believed to be the cause of multi-organ dysfunction. Furthermore, hypoperfusion, ischemia-reperfusion injury, and neurohumoral activation may lead to organ damage, such as renal injury (29). Numerous studies and reports have documented prolonged $\mathrm{CPB}$ duration as an independent risk factor for postoperative AKI in patients with aortic dissection (9,30,31). Xu et al. (9) studied 115 patients who underwent emergent thoracic aortic surgery and found that a 10-minute increase in CPB time was associated with a $17.1 \%$ higher risk for postoperative AKI. Some would argue, however, that the incidence of postoperative AKI was not affected by CPB duration (19,32). These controversial results might be attributed to the different study populations and the confounding factors in heterogeneous patient cohorts. Another reason is that some studies did not include $\mathrm{CPB}$ duration in the multivariate logistic regression equation, although the baseline $\mathrm{CPB}$ duration was higher in patients with AKI than in those without AKI. Therefore, to reduce the duration of $\mathrm{CPB}$, we suggest improvement of the surgical techniques for TAAD and a high degree of anesthesiologist proficiency in CPB.

Subsequently, we investigated the mortality rate of patients with TAAD after cardiac surgery. Our results showed mortality rates of $46.67 \%$ in patients with TAAD and $53.84 \%$ in those who developed AKI. Unfortunately, $66.67 \%$ of AKI patients required CRRT, and their 28day mortality was up to $67.50 \%$. Three large randomized controlled trials on the timing of CRRT in AKI showed that $39.3-58.5 \%$ of AKI patients who received CRRT eventually died (33-35). The results of a retrospective study demonstrated that $58.6 \%$ of patients with cardiac surgery-associated AKI died at 30 days (36). Several studies reported that postoperative AKI itself was an independent predictor of in-hospital mortality after surgery for TAAD and that there was a linear correlation between the AKI severity/stage and mortality $(37,38)$. Another study by Ghoreishi et al. (39) on the preoperative demographics and laboratory values of 269 patients with TAAD showed that lactic acid level, preoperative sCr, and liver malperfusion were the significant independent predictors of postoperative mortality. A similar study by Jiao et al. (40) demonstrated that age $\geq 60$ years, high lactic acid level at 12 hours after CRRT, and long CPB duration were the independent prognostic factors of in-hospital mortality. In this study, there were no independent risk factors for overall postoperative mortality and postoperative mortality in patients with AKI. Also, further analysis showed that preoperative leukocyte level, PEEP, norepinephrine dose upon diagnosis of AKI, CPB duration, and $\triangle \mathrm{CVP}$ in the ICU may be the risk factors for 28-day mortality in postoperative AKI patients who received CRRT. Further analyses identified $\mathrm{CPB}$ duration as the independent risk factor for mortality; this was similar to the results in most of the cases studied. There was a significant difference in CPB duration between the survival and non-survival groups, which implied that longer low-flow, low-pressure, non-pulsatile perfusion with hemodilution and hypothermia may be advantageous. Furthermore, the decrease in CVP during the ICU stay was significantly higher in the survival group than in the nonsurvival group $(\mathrm{P}=0.028)$. Redfors et al. (41) measured the cardiac index (CI), renal blood flow (RBF), and glomerular filtration rate in 12 patients who underwent cardiac surgery and found that the RBF increased as the CI increased.

Norepinephrine dose upon diagnosis of AKI was another independent risk factor for mortality in this study; no similar data were found in previous research. We analyzed 
the results and considered three reasons. Firstly, compared with patients who did not require CRRT, those who needed CRRT had a more clinically severe disease course and more unstable circulation, and thus, required a larger dose of norepinephrine. This point was consistent with a previous view that the mortality rate was higher with more severe disease (38). Secondly, patients in this cohort had severe intravascular volume depletion, as well as insufficient attention and fluid resuscitation. Thirdly, norepinephrine itself may have aggravated renal ischemia and made the kidney injury more serious, which may have led to the increased mortality. A recent study demonstrated that the use of norepinephrine was associated with a relatively high mortality in AKI patients who received CRRT (42). An animal study that evaluated the effects of norepinephrine on kidney circulation in septic AKI found that medullary ischemia and hypoxia were exacerbated after norepinephrine infusion (43). In addition, a high dose of norepinephrine has been associated with an increased mortality due to its catecholamine effects on the cardiovascular system (44). Therefore, the influence of norepinephrine on postoperative AKI patients with TAAD should be considered, as it may induce further deterioration of renal function by increasing renal microvascular resistance and ischemia.

This study had some limitations that should be noted. Firstly, this was a retrospective, single-center, observational study, with relatively few patients. Secondly, this study had some problems in the acquisition of data, such as preand post-operative cardiac function parameters, RBF, fluid management before and during CRRT, as well as changes in the hemodynamics and renal microcirculation. Therefore, we plan to conduct a further prospective multicenter study on this topic.

\section{Conclusions}

The incidence and mortality of postoperative AKI in patients with TAAD in the ICU were higher than those in patients in the department of cardiovascular surgery, with varying and multifactorial risk factors. Preoperative lactic acid level and CPB duration were the independent risk factors for postoperative AKI. CPB duration and norepinephrine dose upon diagnosis of AKI may influence the short-term prognosis of such patients. These results tell us that more attention should be paid to the preoperative tissue perfusion of patients, and more mature surgical procedures may be able to reduce the incidence of postoperative AKI in patients. The use of norepinephrine in critically ill patients who have already developed AKI and transferred to the ICU needs to be more cautious. The results of our study can be used for future research and may contribute to the improvement of patient outcomes.

\section{Acknowledgments}

We are indebted to all the intensive care specialists, study nurses, and patients.

Funding: None.

\section{Footnote}

Reporting Checklist: The authors have completed the STROBE reporting checklist. Available at https://dx.doi. org/10.21037/jtd-21-823

Data Sharing Statement: Available at https://dx.doi. org/10.21037/jtd-21-823

Conflicts of Interest: All authors have completed the ICMJE uniform disclosure form (available at https://dx.doi. org/10.21037/jtd-21-823). The authors have no conflicts of interest to declare.

Ethical Statement: The authors are accountable for all aspects of the work in ensuring that questions related to the accuracy or integrity of any part of the work are appropriately investigated and resolved. This study was approved by Ethics Committee of the Fourth Hospital of Hebei Medical University (2017MEC106), and complied with the Helsinki Declaration guidelines (as revised in 2013). All patients signed the informed consent at admission.

Open Access Statement: This is an Open Access article distributed in accordance with the Creative Commons Attribution-NonCommercial-NoDerivs 4.0 International License (CC BY-NC-ND 4.0), which permits the noncommercial replication and distribution of the article with the strict proviso that no changes or edits are made and the original work is properly cited (including links to both the formal publication through the relevant DOI and the license). See: https://creativecommons.org/licenses/by-nc-nd/4.0/.

\section{References}

1. Hoste EA, Bagshaw SM, Bellomo R, et al. Epidemiology 
of acute kidney injury in critically ill patients: the multinational AKI-EPI study. Intensive Care Med 2015;41:1411-23.

2. Khor CS, Wang WJ. The role of acute kidney injury duration in clinical practice. Ann Transl Med 2019;7(Suppl 3):S88.

3. Kuitunen A, Vento A, Suojaranta-Ylinen R, et al. Acute renal failure after cardiac surgery: evaluation of the RIFLE classification. Ann Thorac Surg 2006;81:542-6.

4. Roh GU, Lee JW, Nam SB, et al. Incidence and risk factors of acute kidney injury after thoracic aortic surgery for acute dissection. Ann Thorac Surg 2012;94:766-71.

5. Aittokallio J, Uusalo P, Kallioinen M, et al. Markers of Poor Prognosis in Patients Requiring Continuous Renal Replacement Therapy After Cardiac Surgery. J Cardiothorac Vasc Anesth 2020;34:3329-35.

6. Lu Y, Xue Y, Zhang H, et al. Management strategy of Type A Aortic Dissection in a developing center from China: 16 years experiences. J Thorac Dis 2020;12:6780-8.

7. Vincent JL, Pelosi P, Pearse R, et al. Perioperative cardiovascular monitoring of high-risk patients: a consensus of 12. Crit Care 2015;19:224.

8. Wang J, Yu W, Zhai G, et al. Independent risk factors for postoperative AKI and the impact of the AKI on 30-day postoperative outcomes in patients with type A acute aortic dissection: an updated meta-analysis and meta-regression. J Thorac Dis 2018;10:2590-8.

9. Xu S, Liu J, Li L, et al. Cardiopulmonary bypass time is an independent risk factor for acute kidney injury in emergent thoracic aortic surgery: a retrospective cohort study. J Cardiothorac Surg 2019;14:90.

10. Schoenrath F, Laber R, Maralushaj M, et al. Survival, Neurologic Injury, and Kidney Function after Surgery for Acute Type A Aortic Dissection. Thorac Cardiovasc Surg 2016;64:100-7.

11. Nadim MK, Forni LG, Bihorac A, et al. Cardiac and Vascular Surgery-Associated Acute Kidney Injury: The 20th International Consensus Conference of the ADQI (Acute Disease Quality Initiative) Group. J Am Heart Assoc 2018;7:e008834.

12. Hansen MK, Gammelager H, Mikkelsen MM, et al. Postoperative acute kidney injury and five-year risk of death, myocardial infarction, and stroke among elective cardiac surgical patients: a cohort study. Crit Care 2013;17:R292.

13. Truche AS, Darmon M, Bailly S, et al. Continuous renal replacement therapy versus intermittent hemodialysis in intensive care patients: impact on mortality and renal recovery. Intensive Care Med 2016;42:1408-17.
14. Ronco C. Continuous renal replacement therapy: fortyyear anniversary. Int J Artif Organs 2017;40:257-64.

15. Schefold JC, von Haehling S, Pschowski R, et al. The effect of continuous versus intermittent renal replacement therapy on the outcome of critically ill patients with acute renal failure (CONVINT): a prospective randomized controlled trial. Crit Care 2014;18:R11.

16. Metnitz PG, Krenn CG, Steltzer H, et al. Effect of acute renal failure requiring renal replacement therapy on outcome in critically ill patients. Crit Care Med 2002;30:2051-8.

17. Uchino S, Bellomo R, Morimatsu H, et al. Continuous renal replacement therapy: a worldwide practice survey. The beginning and ending supportive therapy for the kidney (B.E.S.T. kidney) investigators. Intensive Care Med 2007;33:1563-70.

18. Ranucci M, Aloisio T, Cazzaniga A, et al. Validation of renal-risk models for the prediction of non-renal replacement therapy cardiac surgery-associated acute kidney injury. Int J Cardiol 2018;272:49-53.

19. Kowalik MM, Lango R, Klajbor K, et al. Incidenceand mortality-related risk factors of acute kidney injury requiring hemofiltration treatment in patients undergoing cardiac surgery: a single-center 6-year experience. J Cardiothorac Vasc Anesth 2011;25:619-24.

20. Ma WG, Zheng J, Liu YM, et al. Dr. Sun's Procedure for Type A Aortic Dissection: Total Arch Replacement Using Tetrafurcate Graft With Stented Elephant Trunk Implantation. Aorta (Stamford) 2013;1:59-64.

21. Khwaja A. KDIGO clinical practice guidelines for acute kidney injury. Nephron Clin Pract 2012;120:c179-84.

22. Levey AS, Bosch JP, Lewis JB, et al. A more accurate method to estimate glomerular filtration rate from serum creatinine: a new prediction equation. Modification of Diet in Renal Disease Study Group. Ann Intern Med 1999;130:461-70.

23. Zhao H, Pan X, Gong Z, et al. Risk factors for acute kidney injury in overweight patients with acute type A aortic dissection: a retrospective study. J Thorac Dis 2015;7:1385-90.

24. Apaydin AZ, Buket S, Posacioglu H, et al. Perioperative risk factors for mortality in patients with acute type A aortic dissection. Ann Thorac Surg 2002;74:2034-9; discussion 2039.

25. Englberger L, Suri RM, Greason KL, et al. Deep hypothermic circulatory arrest is not a risk factor for acute kidney injury in thoracic aortic surgery. $\mathrm{J}$ Thorac Cardiovasc Surg 2011;141:552-8. 
26. O'Neal JB, Shaw AD, Billings FT 4th. Acute kidney injury following cardiac surgery: current understanding and future directions. Crit Care 2016;20:187.

27. Borst HG, Laas J, Heinemann M. Type A aortic dissection: diagnosis and management of malperfusion phenomena. Semin Thorac Cardiovasc Surg 1991;3:238-41.

28. Fann JI, Sarris GE, Mitchell RS, et al. Treatment of patients with aortic dissection presenting with peripheral vascular complications. Ann Surg 1990;212:705-13.

29. Wang Y, Bellomo R. Cardiac surgery-associated acute kidney injury: risk factors, pathophysiology and treatment. Nat Rev Nephrol 2017;13:697-711.

30. Arnaoutakis GJ, Vallabhajosyula P, Bavaria JE, et al. The Impact of Deep Versus Moderate Hypothermia on Postoperative Kidney Function After Elective Aortic Hemiarch Repair. Ann Thorac Surg 2016;102:1313-21.

31. O'Sullivan KE, Byrne JS, Hudson A, et al. The effect of obesity on acute kidney injury after cardiac surgery. J Thorac Cardiovasc Surg 2015;150:1622-8.

32. Kim WH, Lee SM, Choi JW, et al. Simplified clinical risk score to predict acute kidney injury after aortic surgery. J Cardiothorac Vasc Anesth 2013;27:1158-66.

33. Zarbock A, Kellum JA, Schmidt C, et al. Effect of Early vs Delayed Initiation of Renal Replacement Therapy on Mortality in Critically Ill Patients With Acute Kidney Injury: The ELAIN Randomized Clinical Trial. JAMA 2016;315:2190-9.

34. Gaudry S, Hajage D, Schortgen F, et al. Initiation Strategies for Renal-Replacement Therapy in the Intensive Care Unit. N Engl J Med 2016;375:122-33.

35. Barbar SD, Clere-Jehl R, Bourredjem A, et al. Timing of Renal-Replacement Therapy in Patients with Acute Kidney Injury and Sepsis. N Engl J Med 2018;379:1431-42.

36. Wu B, Sun J, Liu S, et al. Relationship among Mortality of Patients with Acute Kidney Injury after Cardiac Surgery, Fluid Balance and Ultrafiltration of Renal

Cite this article as: Zhang K, Shang J, Chen Y, Huo Y, Li B, $\mathrm{Hu} \mathrm{Z}$. The prognosis and risk factors for acute kidney injury in high-risk patients after surgery for type A aortic dissection in the ICU. J Thorac Dis 2021;13(7):4427-4437. doi: 10.21037/jtd21-823
Replacement Therapy: An Observational Study. Blood Purif 2017;44:32-9.

37. Olsson C, Franco-Cereceda A. Impact of organ failure and major complications on outcome in acute Type A aortic dissection. Scand Cardiovasc J 2013;47:352-8.

38. Sasabuchi Y, Kimura N, Shiotsuka J, et al. Long-Term Survival in Patients With Acute Kidney Injury After Acute Type A Aortic Dissection Repair. Ann Thorac Surg 2016;102:2003-9.

39. Ghoreishi M, Wise ES, Croal-Abrahams L, et al. A Novel Risk Score Predicts Operative Mortality After Acute Type A Aortic Dissection Repair. Ann Thorac Surg 2018;106:1759-66.

40. Jiao R, Liu N. Prognostic factors for in-hospital mortality in patients with acute kidney injury requiring continuous renal replacement therapy undergoing surgery for acute Stanford type A aortic dissection. Zhonghua Wai Ke Za Zhi 2017;55:270-3.

41. Redfors B, Bragadottir G, Sellgren J, et al. Effects of norepinephrine on renal perfusion, filtration and oxygenation in vasodilatory shock and acute kidney injury. Intensive Care Med 2011;37:60-7.

42. Chen YY, Wu VC, Huang WC, et al. Norepinephrine Administration Is Associated with Higher Mortality in Dialysis Requiring Acute Kidney Injury Patients with Septic Shock. J Clin Med 2018;7:274.

43. Lankadeva YR, Kosaka J, Evans RG, et al. Intrarenal and urinary oxygenation during norepinephrine resuscitation in ovine septic acute kidney injury. Kidney Int 2016;90:100-8.

44. Levy B, Dusang B, Annane D, et al. Cardiovascular response to dopamine and early prediction of outcome in septic shock: a prospective multiple-center study. Crit Care Med 2005;33:2172-7.

(English Language Editor: A. Kassem) 